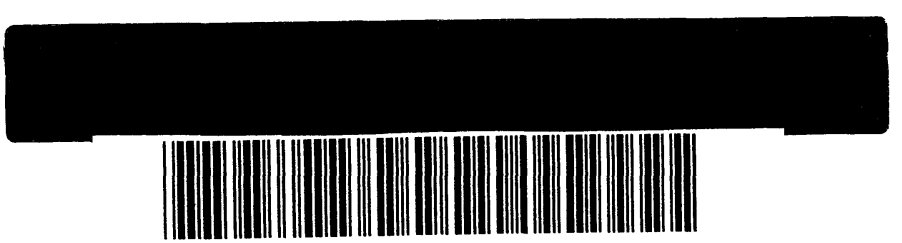

CM-P00052017

\title{
DIFFERENTIAL ALGEBRAS IN FIELD THEORY
}

by

R. Stora

LAPP, BP.909, F-74019 Annecy-le-Vieux

and

Theory Division, CERN, CH-1211 Geneva 23

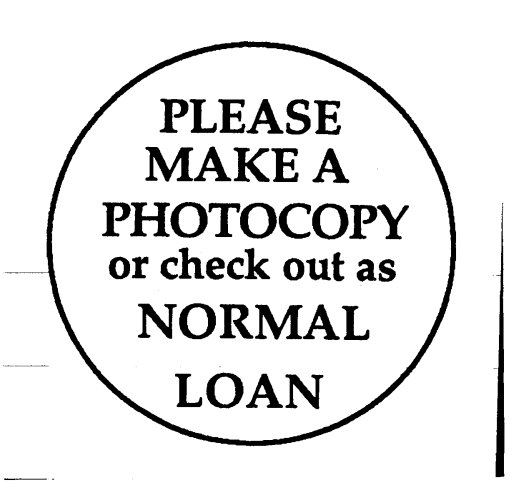

Talk given at the Second Summer Meeting on "Quantum Mechanics of Fundamental Systems" Dec. 17-20, 1987, Santiago, Chile 


\section{INTRODUCTION}

Differential algebras have been known for a long time to be mathematical tools appropriate to field theory [cf., e.g., [1]]. Classically, the de Rham algebra of differential forms on the space time manifold is favoured. More exotic differential algebras made their appearance some fifteen years ago, in the context of perturbatively quantized gauge theories and by now belong to the standard equipment of field theorists as either necessary or only convenient tools, to deal with gauge theories of rather general types. It is easier to talk about these things now than ten of fifteen years ago because many people are not so allergic anymore to the mathematics of the fifties as they used to be. Preparing these notes served as an opportunity to recollect some memories and dig out some documents which were not widely circulated. I have not attempted at any completeness, not only because it is very difficult, possibly impossible, given the abundance of literature on the subject, but also because of personal prejudices which I shall try to justify.

The exotic example at the start of this industry has to do with the Slavnov[3] symmetry of the Faddeev Popov[2] gauge fixed Yang Mills theory whose main property is to manifestly maintain locality throughout the renormalization procedure. This example has served as a prototype for a number of extensions. Among the best developed and most remarkable is the analysis of constrained Hamiltonian systems developed by the Lebedev group[4]. I will not touch this body of knowledge, because, in spite of its recent popularity in string theory, it fails to accomodate simultaneously the locality of field theory and geometry, which the original Lagrangian version allows to do. It has however the advantage to allow for a Hilbert space framework. Actually the operator $\mathbf{Q}$, as well as $\bar{Q}$, popular in string theory[5] and related to the conformal structure of the theory, and which bear some formal similarity to that of the Lebedev construction[4] in the particular case where the constraints generate a Lie algebra, is nothing else than the coboundary operator for the cohomology of the Virasoro algebra via a formula due to J.L. Koszul[6]. At the Lagrangian level, solving specific examples shows that a general solution is missing for what may be called the auxiliary field problem"). Unfortunately most known examples result into more or less trivial extensions of the cohomology algebra of some (gauge) Lie algebra. Even there, the auxiliary field problem has only been solved in particular cases e.g. in $\mathrm{N}=1$ supergravities where the solution is not unique [28]. The standard way out[10][14][29] is to introduce external fields, but the correct definition of physical observables is even more conjectural. Apart from

*) i.e. can a non integrable situation be viewed as a restriction of an integrable one. 
these classes of examples two others are instructing by themselves : the Torino group manifold approach[7] which grounds the construction of geometrical theories (e.g. supergravities) on generalized Weyl algebras; the other prototype example is that of quantized p-differential forms[8]. Much of the present activity is concerned with applications to string theory, first and second quantized. I trust this area will be covered by other speakers at this conference. I will therefore limit myself to a discussion of the principles which may be abstracted from the examples I know best.

Section II will recall the situation in the Yang Mills theories and expound a parallel treatment of the first quantized bosonic string.

Section III is devoted to a sketchy account of a nice by-product : the algebraic description of anomalies.

Section IV poses a general class of problems suggested by the examples of Section 2.

\section{YANG MILLS THEORIES AND FIRST OUANTIZED STRINGS}

\section{Yang Mills Theories}

Let us recall that the Faddeev Popov gauge fixed action, in a Feynman like gauge, reads:

$$
S(a, \omega, \vec{\omega})=S_{i n v}(a)+S_{g f} ; S_{g f}=\int_{M^{4}}\left[\frac{1}{2}\{g, g\}+\{\bar{\omega}, M \omega\}\right] d^{4} x,
$$

where $a$ is the Yang Mills field

$$
a=\sum_{\mu=0 \ldots 3} a_{\mu}^{\alpha}(x) d x^{\mu} e_{\alpha}
$$

$\alpha$

$e_{\alpha}$ : a basis of Lie $G$, the Lie algebra of the compact group $G$, with commutation relations

$$
\left[e_{\alpha}, e_{\beta}\right]=f_{\alpha \beta}^{\gamma} e_{\gamma}
$$




$$
\begin{array}{r}
F(a)=d a+\frac{1}{2}[a, a]=\frac{1}{2} \sum_{\mu, v} F_{\mu v} d x^{\mu} \wedge d x^{v} \\
=\frac{1}{2} \sum_{\mu, v} F_{\mu v}^{\alpha} d x^{\mu} \wedge d x^{v} e_{\alpha} \\
F_{\mu v}^{\alpha}=\partial_{\mu} a_{v}^{\alpha}-\partial_{v \mu} a_{\mu}^{\alpha}+f_{\beta \gamma}^{\alpha} a_{\mu}^{\beta} a_{v}^{\gamma} \\
S_{\text {inv }}(a)=-\frac{1}{4} \int d^{4} x\left(F_{\mu v}, F^{\mu v}\right)
\end{array}
$$

where $($,$) is a G$ invariant bilinear form on Lie $G g$ is a Lie $G$ valued ${ }^{*}$ ) local function of a (and its derivatives), $\{$,$\} another quadratic form on Lie G$ (e.g. $\left.g^{\alpha}(x)=\partial_{\mu} a^{\mu \alpha}(x)+\ldots\right)$

$$
\omega=\sum_{\alpha}^{\alpha} \mathrm{e}_{\alpha}
$$

is the Faddeev Popov geometrical ghost which serves as a generator of the Grassmann algebra of the dual of Lie $\mathcal{G}$, the Lie algebra of the gauge group $\mathcal{G}$ :

$$
\mathcal{G}=\left\{\text { Maps } \mathrm{M}_{4} \rightarrow \mathrm{G}\right\}
$$

with the obvious pointwise composition rules. If $\Lambda \in$ Lie $G$ is represented by:

$$
\begin{aligned}
& \Lambda_{x}=\sum_{d} \Lambda^{\alpha}(x) e_{\alpha} . \\
& \left\langle\omega^{\alpha}(x), \Lambda>=\Lambda^{\alpha}(x) .\right.
\end{aligned}
$$

$M \omega$ is the variation of $g$ under an operation of Lie $G$, of parameter $\omega$, induced by:

$$
\delta_{\Lambda} a=d \Lambda+[a, \Lambda]
$$

\footnotetext{
*) This is a convenient labelling of the set of gauge functions whose number is $\operatorname{dim} G$, but it is also misleading: the geometrical nature of $g$ need not in principle have anything to do with Lie G. This will become apparent e.g. in the string case (section 2).
} 
$\bar{\omega}$, the Faddeev Popov antighost, also a Grassmann type field is a Lagrange multiplier which, in the present instance generates another copy of $\Lambda$ Lie $G^{*}$ ). This is a peculiarity of this example as we shall see that, in general $\bar{\omega}$ has geometrical properties which depend on those of the gauge function.

The full action $S$ is found to be invariant under the Slavnov symmetry[3]:

$$
\begin{aligned}
& s a=-d \omega-[a, \omega] \\
& s \omega=-\frac{1}{2}[\omega, \omega] \\
& s \vec{\omega}=-g .
\end{aligned}
$$

This was derived in two steps : using a beautiful trick"*) due to A.A. Slavnov[3] one first derives the Slavnov identity for, say, the connected Green functional[9]; then one recognizes that the Slavnov identity expresses the invariance of the action under the Slavnov symmetry Eq. $(10)^{[10]}$.

In the initial version, the transformation contained an anticommuting infinitesimal parameter which was soon gotten rid of [11], as above, considering $s$ as a graded derivation on the algebra of functionals of $a, \omega, \vec{\omega}$ - the total grading being given by $\# \omega-\# \vec{\omega}$ (+ form degree, if one anticipates section III).

Also note that in the form given in eq. (10) one does not have $s^{2}=0$ because

$$
\mathrm{s}^{2} \bar{\omega}=-M \omega
$$

i.e. it is only nilpotent modulo the ghost equation of motion. This phenomenon which creates mathematical inconveniences will be repeatedly met. Here it is easily eliminated by the introduction of a multiplier field $b$, turning $S_{g f}$ into

$$
\mathrm{S}_{\mathrm{gf}}(\mathrm{a}, \omega, \vec{\omega} \mathrm{b})=\int \mathrm{b}_{\alpha}(\mathrm{x}) g^{\alpha}(\mathrm{x}) \mathrm{dx}+\int \vec{\omega}_{\alpha} M_{\beta}^{\alpha} \omega^{\beta} \mathrm{dx}+F(\mathrm{~b})
$$

\footnotetext{
") See however the remark in the previous footnote.

**) Write the Ward identity which characterizes the breaking of gauge invariance of the total action, only gauge transforming the gauge field, and use as an infinitesimal gauge parameter $\Lambda=M^{-1} \omega$.
} 
invariant under

$$
\begin{aligned}
& s \omega_{\alpha}=-b_{\alpha} \\
& s b_{\alpha}=0,
\end{aligned}
$$

so that $\mathcal{F}(b)$ can be chosen as a quadratic functional reproducing the initial action upon elimination of the b-field - whereby nilpotency of $s$ is lost. I have always called $b$ the Stueckelberg multiplier because it was very similar to the ghost field introduced by Stueckelberg to remedy the non renormalizability of massive QED by passing to the Stueckelberg gauge[12], but this multiplier field is commonly called the Nakanishi Lautrup field.

This algorithm can be generalized to allow the metric $\{$,$\} in Eq.(1) to be$ field - a - dependent at the expense of introducing an extra ghost[13].

In order to write down properly the Slavnov identity, it is convenient to add to the action a source term

$$
\mathrm{S}_{\text {source }}=\int A \mathrm{sa}+\Omega \mathrm{s} \omega
$$

so that

$$
S_{\text {tot }}=S_{\text {inv }}+S_{g f}+S_{\text {source }}
$$

fulfills:

$$
\int\left(\frac{\delta S_{\text {tot }}}{\delta A} \frac{\delta S_{\text {tot }}}{\delta a}+\frac{\delta S_{\text {tot }}}{\delta \Omega} \frac{\delta S_{\text {tot }}}{\delta \omega}-b \frac{\delta S_{\text {tot }}}{\delta \vec{\omega}}\right)=0
$$

which "easily" passes through renormalization - modulo anomalies, cf. section III by substituting the vertex functional $\Gamma$ against $S_{\text {tot. }}$.

Legendre transforming wr. to $b^{*}$ ) and calling $\Omega$ the Legendre transform yields for the Legendre transform $\tilde{S}$ of $S$ the more symmetrical identity[14]*)

*) Another way to construct $\tilde{S}$ is to argue that the symmetry of $S_{\text {inv. }}+S_{g f}+S_{\text {source }}$ is best expressed by adding a term $\int \Omega g$, so that the resulting action, $\Sigma$ fulfills 


$$
\int \frac{\delta \tilde{S}}{\delta A} \frac{\delta \tilde{S}}{\delta \mathrm{a}}+\frac{\delta \tilde{S}}{\delta \Omega} \frac{\delta \tilde{S}}{\delta \omega}+\frac{\delta \tilde{S}}{\delta \bar{\Omega}} \frac{\delta \tilde{S}}{\delta \bar{\omega}}=0
$$

Whereas $\mathrm{b}$ has to be considered as a quantum field, $\bar{\Omega}$ has to be considered as a classical field serving as a source of the gauge function $\left(S_{\text {gf }}\right.$ now gets a term $1 / 2(g-\bar{\Omega}, g-\bar{\Omega})+\vec{\omega} M \omega$. The Slavnov identity encodes two symmetries of the action:

$$
\begin{aligned}
& s \vec{\omega}=0 \\
& s \vec{\Omega}=M \omega=s g
\end{aligned}
$$

which is nilpotent,

$$
\begin{aligned}
& s \bar{\omega}=-(\boldsymbol{g}-\bar{\Omega}) \\
& s \vec{\Omega}=0
\end{aligned}
$$

which is not.

The Slavnov operator is defined as

$$
\mathcal{J}=\int \frac{\delta \tilde{\mathrm{S}}}{\delta A} \frac{\delta}{\delta \mathrm{a}}+\frac{\delta \tilde{\mathrm{S}}}{\delta \mathrm{a}} \frac{\delta}{\delta A}+\frac{\delta \tilde{\mathrm{S}}}{\delta \Omega} \frac{\delta}{\delta \omega}+\frac{\delta \tilde{\mathrm{S}}}{\delta \omega} \frac{\delta}{\delta \Omega}+\frac{\delta \tilde{\mathrm{S}}}{\delta \vec{\Omega}} \frac{\delta}{\delta \bar{\omega}}+\frac{\delta \tilde{S}}{\delta \bar{\omega}} \frac{\delta}{\delta \bar{\Omega}}
$$

and $\mathbf{8}^{2}=0$ follows from Eq.(16). This is the important operator in perturbation theory. The discussion of anomalies i.e. the appearance of a right hand side in Eq.(16), when $S$ is replaced by $\Gamma$ goes through the discussion of local functionals $\int \alpha$, which fulfill the Wess Zumino[15] consistency condition - actually a generalization thereof:

$$
\int \frac{\delta \Sigma}{\delta A} \frac{\delta \Sigma}{\delta a}+\frac{\delta \Sigma}{\delta \Omega} \frac{\delta \Sigma}{\delta \omega}+\frac{\delta \Sigma}{\delta \bar{\Omega}} \frac{\delta \Sigma}{\delta g}=\Omega \frac{\delta \Sigma}{\delta \omega} ; \text { then } \tilde{S}=\Sigma-\frac{\bar{\Omega}^{2}}{2} .
$$




$$
\rho \int_{M^{4}} \alpha=0
$$

modulo trivial anomalies of the form

$$
\int \alpha=\$ \Gamma^{\text {loc }}
$$

where $\Gamma^{\text {loc }}$ is a local functional.

The nice thing[11][16] is that the only non trivial part of this cohomology involves $a$ 's which are local in $a, \omega$ and fulfill

$$
s \int \alpha(\omega, a)=0
$$

jacdefined modulo s $\Gamma^{\text {loc }}(a, \omega)$.

which takes us back to the conventional Wess Zumino consistency conditions[15]. Given the Slavnov identity, one can prove gauge independence and unitarity whenever an asymptotic theory exists[10]. The definition of asymptotic physical states has been nicely algebraized by T. Kugo and I. Ojima[17], using the b-field. This goes through the construction of the Noether current associated with the Slavnov symmetry and the definition of the corresponding asymptotic charge $Q$ which is nilpotent, and commutes with the $S$ operator if there is no anomaly. Physical states are then defined by the cohomology of $Q$ for zero ghost number. The Noether current algebra is interesting in itself[18], although it is not unique because of the occurence of derivatives in the transformation laws.

\section{The free bosonic string}

The free bosonic string can be treated in a way parallel to Yang Mills theories with a few delicacies. The invariant action is 
$X=(\sigma, \tau) \in \Sigma$ parametrizing a world sheet, $X \in R^{D}$, the Euclidean metric in $R^{\left.D^{*}\right)}$, g a metric on $\Sigma$.

One may take two attitudes. Either one has $S_{\text {inv }}$ depend on $g^{[19]}$. Then $S_{\text {inv }}$ is invariant under Diff $\Sigma \times$ Weyl, where Weyl transformations locally scale $g$. One has to pick three gauge functions which one may choose as the three components of $\mathrm{g}$ and the full Slavnov symmetry results as a current algebra Ward identity for the stress energy tensor coupled to $\mathrm{g}$, the multiplier field being trivially eliminated in a Landau type gauge $(F(b)=0$ in eq.(1')).

Or one may have $S_{\text {inv }}$ only depend on the conformal class of $\mathrm{g}^{[20]}$ and choose the gauge fixing in such a way that the conformal structure of the theory is manifestly exhibited[5].

Now, one knows a nice parametrization of conformal classes of metrics, in terms of Beltrami differentials : picking a reference complex structure with local complex analytic coordinates $\mathbf{z , z}$, the conformal class is characterized by

$$
\mathrm{ds}^{2} \propto \operatorname{ldz}+\left.\mu \mathrm{dz}\right|^{2} \quad|\mu|<1
$$

Then, in terms of $\mu, \mu, S_{\text {inv }}$ reads

$$
S_{\text {inv }}=\int \frac{d z \wedge d \bar{z}}{2 i} \frac{1}{1-\mu \bar{\mu}}\left(\partial_{z}-\bar{\mu} \partial_{\bar{z}} X, \partial_{z}-\mu \partial_{z} X\right)
$$

C. Becchi has found that the unique choice of gauge functions which exhibits the theory as a conformal theory is the pair $\mu-\mu^{0}, \bar{\mu}-\bar{\mu}^{\left.0^{* *}\right)}$. Eliminating the multiplier field yields the gauge fixed action:

$$
S=S_{\text {inv }}+S_{g f}
$$

\footnotetext{
") we use the euclidean version throughout although locality does not care.

**) The easiest way is to proceed via the elimination of the b field as well as of the Weyl ghost and antighost. One may also start directly with conformal classes of metrics, eliminate the $b$ field a la Kato Ogawa[20],[5] introduce a source $\mu, \bar{\mu}$ coupled to the s-variation of the antighost, and recover a Slavnov identity a la Zinn Justin (C.Becchi, private communication).
} 


$$
\begin{aligned}
& S=S_{\text {inv }}+S_{g f} \\
& S_{g f}=\int \frac{d z \wedge d \vec{z}}{2 i} b_{z z}\left(\partial_{\vec{z}}-\mu \partial_{z}+\partial_{z} \mu\right) C^{z}+c . c .
\end{aligned}
$$

Here the ghost $C^{z}$ is related to the diffeomorphism ghost $\xi=\left(\xi^{z}, \xi^{z}\right)$ through

$$
C^{z}=\xi^{z}+\mu \xi^{z} \& \text { c.c. }
$$

and the Slavnov symmetry associated with diffeomorphisms reads

$$
\begin{aligned}
& s X=(\xi . \partial) X=\left(\xi z \partial_{z}+\xi \bar{z} \partial_{z}\right) X \\
& s C^{z}=C^{z} \partial_{z} C^{z} \& c . c . \\
& \text { or } s \xi=(\xi . \partial) \xi \\
& s \mu=\partial_{z} C^{z}+C^{z} \partial_{z} \mu-\mu \partial_{z} C^{z} \\
& s b_{z z}=0
\end{aligned}
$$

The corresponding Slavnov identity defines the current algebra for the two components $\Theta_{\mathrm{zz}}, \Theta_{\overline{\mathrm{z}} \overline{\mathrm{z}}}$ of the stress energy tensor, whose correlation functions are obtained through functional differenciation with respect to $\mu, \bar{\mu}$.

The conformal properties of the system are furthermore characterized by a set of Ward identities which assign conformal weights $0,-1,+2$ to $X, C z, b_{z z}$, namely, the action is invariant under the following action of diffeomorphisms**) :

$$
\begin{aligned}
& \delta_{\lambda} X=(\lambda . \partial) X \\
& \delta \lambda \mu=\partial_{z} \Lambda+\Lambda \partial_{z} \mu-\mu \partial_{z} \Lambda \\
& \left.\quad \stackrel{\text { def }}{=} \lambda^{z}+\mu \lambda^{\bar{z}}\right) \\
& \delta_{\lambda} C^{z}=(\lambda . \partial) C^{z}-\left(\partial_{z} \lambda^{z}+\mu \partial_{z} \lambda \vec{z}\right) C z \\
& \delta_{\lambda} b_{z z}=(\lambda . \partial) b_{z z}+2\left(\partial_{z} \lambda z+\mu \partial_{z} \lambda^{z}\right) C^{z}
\end{aligned}
$$

\footnotetext{
*) Note that the geometrical nature of the antighosts $b_{z z}, b_{z z}$ is related to that of the gauge function as mentioned in II.1.

**) L. Baulieu, M. Bellon[20][21] have found another action of diffeomorphisms which leaves $S$ invariant but does not apply to more general conformal models involving Thirring like couplings.
} 
The action defines Feynman rules which entails factorization of the Green functions into factors involving $z$ indices alone, and factors involving $\bar{z}$ indices alone. The anomalies are easily computed and of course found to be proportional to D-26") .

As a side remark, transformations (29) apply to any conformal model i.e. Lagrangian model which one can couple to a conformal class of metrics - or complex structure - represented by $\mu$ : for a field $\varphi_{q} \bar{q}$ with conformal weights $q, \bar{q}$ one finds

$$
\delta_{\lambda} \varphi_{\mathrm{qq}}=\left[(\lambda . \partial)+\mathrm{q}\left(\partial_{\mathrm{z}} \lambda^{\mathrm{z}}+\mu \partial_{\mathrm{z}} \lambda^{\overline{\mathrm{z}}}\right)+\overline{\mathrm{q}}\left(\partial_{\overline{\mathrm{z}}} \lambda^{\overline{\mathrm{z}}}+\bar{\mu} \partial_{\overline{\mathrm{z}}} \lambda^{\mathrm{z}}\right)\right] \varphi_{\mathrm{qq}}
$$

which checks with eq. $(29)$ for $(q, \bar{q})=(0,0),(-1,0),(+2,0)$, \& c.c. for $X, c^{z}, b_{z z}$.

The whole scheme can be extended to include the Noether current algebra of the Slavnov symmetry[21]. Just as in the Yang Mills case the anomaly is rigidly tied up with the "holomorphy anomaly" ( $\propto D-26)$ and yields a local analog of the Kato Ogawa nilpotency anomaly for the charge.

Another similarity with the Yang Mills case is the form of the Slavnov identity: introducing

$$
s_{\text {source }}=\int \varkappa s X+\varphi s C^{2}+c . c .
$$

we have for

$$
\begin{aligned}
& S_{\text {tot }}=S_{\text {inv }}+S_{g f}+S_{\text {source }} \\
& \int \frac{d z \wedge d \bar{z}}{2 i}\left(\frac{\delta S}{\delta x} \frac{\delta S}{\delta X}+\frac{\delta S}{\delta e} \frac{\delta S}{\delta C^{z}}+\frac{\delta S}{\delta b_{z z}} \frac{\delta S}{\delta \mu}+\text { c.c. }\right)=0 .
\end{aligned}
$$

*) Their algebraic form reads

$\int \frac{d z \wedge d \bar{z}}{2 i}\left[\Lambda^{z} \partial_{z}^{3} \mu-\mu \partial_{z}^{3} \Lambda^{z}+R\left(\Lambda^{z} \partial_{z} \mu-\mu \partial_{z} \Lambda^{z}\right)\right]+c . c$.

where $R$ is a projective connection[30]. 
The last term may be interpreted in two ways: the invariance of $S$ under (28), which is nilpotent, or under

$$
\begin{aligned}
& s^{\prime} \mu=0 \\
& s^{\prime} b_{z z}=\frac{\delta S}{\delta \mu}=\Theta_{z z}
\end{aligned}
$$

\& c.c.

which is only nilpotent modulo the ghost equations of motion[5]. The existence of the Slavnov identity can be however most simply obtained via the existence of the nilpotent transformation (28).

A last remark is in order : the conformal structure of the model is obtained at the expense of having chosen a gauge function which is not in general a good gauge function - unless there is only one orbit of $\mu$ 's under diffeomorphisms. For instance, if $\Sigma$ is a compact Riemann surface of genus $g>1$, there are $3 g-g$ zero modes for $b_{z z}$ to be gauge fixed [the analogue of the Gribov horizon in the Y.M. case]. We shall return to this problem in section III.

\section{IHEALGEBRAIC STRUCTURE OE LOCALANOMALIES}

We have mentioned in section II the possible occurence at the quantum level of local anomalies fulfilling the proper Wess Zumino consistency condition, and mentioned that only the dual Lie algebra part of the Slavnov symmetry was involved. In the Yang Mills case, the $\$$ operation when restricted to a and $\omega$ reduces[22] to the coboundary operator defining the local cohomology of the gauge Lie algebra with values in the algebra of local functionals of a.

This allows to give compact formulae for the Adler Bardeen anomalies derived e.g. in ref. [16] and this has turned into a small industry a few years ago[23]. This is a subject of its own for which we have indicated[23] a few references which allow to trace back most of the literature. The temperature has now decreased on this topic, but there is still one interesting mathematical problem to be settled. There are in fact two theories of anomalies. An algebraic one, the "local" theory sketched out here, which reduces the problem in each model to computing a set of numerical coefficients, but in particular does not explain the rationality of various coefficients at the one loop level, a property which is stable thanks to the non renormalizability 
theorems a la Bardeen. Nor does it give any clue to why it only occurs when chiral fields are involved. But this explains $\pi^{\circ} \rightarrow 2 \gamma$.

On the other hand, there is the topological theory[23][24] due to Atiyah Bott Singer Quillen and others, which is purely euclidean as it deals with the Index of families of elliptic operators and relates anomalies to de Rham cohomology classes of e.g. gauge groups. The puzzle is that the local anomaly responsible for $\pi^{\circ} \rightarrow 2 \gamma$ corresponds to a trivial de Rham cohomology class, a fact which was known to physicists[25]. This phenomenon presumably also happens in the case of gravitational anomalies. This situation raises an interesting mathematical problem : can one construct an Index theory in terms of local cohomology classes "on the fibers"?

Besides, on the local side, there is still much to be done. Work is still in progress[26] and progress is definitely needed.

\section{LESSONS TOBE DRAWN}

The examples of section II pose the problem of quantizing a degenerate classical action. This means that $S(\varphi), \varphi=\left\{\varphi^{i}\right\}$, is invariant under some transformations

$$
\delta_{\omega} \varphi=P(\varphi, \omega)
$$

where $P(\varphi, \omega)$ is linear in $\omega=\left\{\omega^{\alpha}\right\}$. We are mostly concerned with local actions and local transformations. Note that any action is invariant under uninteresting transformations

$$
\delta \varphi^{i}=M^{i j} \frac{\delta S}{\delta \varphi^{j}}
$$

where $M^{i j}$ is a (graded) symmetric kernel. If the transformations (34) are integrable i.e. express differenciation along the leaves of a foliation in field space, they give rise to a nilpotent Slavnov symmetry $s$ which is nothing but differenciation along the leaves. By a formal Frobenius property, if the operations $\delta_{\omega}$ are in involution, namely 


$$
\left[\int \delta_{\omega_{1}} \varphi \frac{\delta}{\delta \varphi}, \int \delta_{\omega_{2}} \varphi \frac{\delta}{\delta \varphi}\right]=\int \delta_{\mathrm{f}\left(\omega_{1}, \omega_{2}, \varphi\right)} \varphi \frac{\delta}{\delta \varphi}
$$

with $f$ antisymmetric in $\omega, \omega_{2}$, there will be a foliation, locally and therefore an $S$ operation"):

$$
\begin{aligned}
& s \varphi=P(\varphi, \omega) \\
& s \omega=-\frac{1}{2} f(\omega, \omega, \varphi)
\end{aligned}
$$

$s^{2}=0$ is a consequence of the Jacobi identity.

This situation occurs not only when a Lie algebra is involved but for instance genuinely to define $\sigma$-models[27] where one has to express that splitting the field into a background and fluctuations is invariant under an infinitesimal change of the background and an adequately corresponding change of the fluctuating part.

A more general situation which occurs frequently, and which we have already met is when closure of the algebra - i.e. nilpotency of the $s$ operation - is only fulfilled modulo the equations of motion i.e. the "uninteresting" operations of eq.(35). This occurs in supergravity where closure can be achieved in a variety of ways via the introduction of either of several possible sets of auxiliary fields[28]. Once closure is achieved gauge fixing can be performed by introducing multiplier fields and antighosts, after choosing a gauge function.

A large amount of work has been performed on the general problem[29].

My present understanding is limited and not quite in agreement with the published literature (mostly the articles by I.A. Batalin, G.A. Vilkovisky). It relies on unpublished work by $M$. Tonin, and generalizes the Yang Mills case treated a la Zinn Justin[14] (eq.16) and the case of the string as treated by $C$. Becchi[20] (eq.32). One assumes $s$ given by eq.(37) with $s^{2}=0$ only modulo the irrelevant transformations eq.(35). One introduces sources $\Phi, \Omega$ coupled to $s \varphi, s \omega$. One may first look for an action $S(\varphi, \omega ; \Phi, \Omega)$ fulfilling

\footnotetext{
") By abuse of notation we denote by $\omega$ the $\phi \pi$ ghost corresponding to the infinitesimal transformations parametrized by $\omega$.
} 


$$
\frac{1}{2}[\underline{S}, \underline{S}] \equiv \int \frac{\delta S}{\delta \varphi} \frac{\delta S}{\delta \Phi}+\frac{\delta S}{\delta \omega} \frac{\delta S}{\delta \Omega}=0
$$

Or one may directly look for $S(\varphi, \omega, \omega ; \Phi \Omega \Omega)$ such that

$$
\frac{1}{2}[\underline{S}, \underline{S}] \equiv \int \frac{\delta S}{\delta \varphi} \frac{\delta S}{\delta \Phi}+\frac{\delta S}{\delta \omega} \frac{\delta S}{\delta \Omega}+\frac{\delta S}{\delta \bar{\Omega}} \frac{\delta S}{\delta \bar{\omega}}=0
$$

$S$ has zero ghost number $(=\# \omega-\# \omega-\# \Phi-2 \# \Omega)$. The notation $[S, S)$ refers to the graded Lie bracket of the vector fields

$$
\underline{S} \equiv \int \frac{\delta S}{\delta \varphi} \frac{\delta}{\delta \Phi}+\frac{\delta S}{\delta \Phi} \frac{\delta}{\delta \varphi}+\frac{\delta S}{\delta \omega} \frac{\delta}{\delta \Omega}+\frac{\delta S}{\delta \Omega} \frac{\delta S}{\delta \omega}+\frac{\delta S}{\delta \bar{\Omega}} \frac{\delta}{\delta \bar{\omega}}+\frac{\delta S}{\delta \bar{\omega}} \frac{\delta}{\delta \bar{\Omega}}
$$

One solves eq.(39) for local functionals with the boundary conditions imposed by the data $S(\varphi), s \varphi, s \omega$. This fixes the Feynman rules for the quantum fields $\varphi, \omega$, $\vec{\omega}$, in presence of the classical fields $\Phi, \Omega, \vec{\Omega}$. One then has to study the quantum extensions $\Gamma$ fulfilling (40), or the occurence of anomalies. Note that the defining equation for $S$ is invariant under the restricted canonical transformations - which do not alter the quantized fields .

$$
\begin{aligned}
& (\varphi, \omega, \bar{\omega}) \rightarrow(\varphi, \omega, \bar{\omega}) \\
& (\Phi, \Omega, \bar{\Omega}) \rightarrow\left(\Phi+\frac{\delta \chi}{\delta \varphi}, \Omega+\frac{\delta \chi}{\delta \omega}, \bar{\Omega}+\frac{\delta \chi}{\delta \bar{\omega}}\right)
\end{aligned}
$$

where $\chi$ is an odd generating function which only depends on $(\varphi, \omega, \bar{\omega})$. This reflects the freedom in the choice of gauge functions. Besides locality, power counting restricts this construction. Since the b-field is absent from this scheme, there does not appear to be a transparent algebraization of the asymptotic theory when it exists, but one may try to construct the current algebra for a Noether current. Observables may be defined in the zero ghost number sector either by the local cohomology of $\underline{S}$ or by the local cohomology of $\underline{S}$ modulo $d$ (d being the exterior derivative). In the Yang Mills case, the first local cohomology is non empty and defines "gauge invariant operators". In the string case - presumably in all cases 
where diffeomorphisms are involved - the physics is presumably concentrated in the local cohomology of $S$ mod $d$, which yields integrated observables. This has to be studied in each case and is really the most important point.

There is one last amusing example with which this section will be concluded: in the treatment of the bosonic string in the conformal gauge, I mentioned the global zero mode problem which occurs because of the bad choice of the gauge function.

The ghost action

$$
S_{g h}=\int \frac{d z \wedge d \vec{z}}{2 i} b_{z z} s \mu=\int b_{z z}\left(\partial_{z} c^{z}+c^{z} \partial_{z} \mu-\mu \partial_{z} c^{z}\right) \frac{d z \wedge d \bar{z}}{2 i}
$$

is invariant under

$$
b_{z z} \rightarrow b_{z z}+\delta b_{z z} \quad \delta b_{z z}=\sum_{i=1}^{3 g-3} c_{i} \phi^{i}
$$

where the $\phi^{i^{\prime}}$ are solution of

$$
\left(-\partial_{\overline{\mathbf{z}}}+\mu \partial_{\mathrm{z}}+2 \partial_{\mathrm{z}} \mu\right) \phi^{\mathrm{i}}=0
$$

i.e. the $\phi^{i}$ 's correspond to holomorphic quadratic differentials for the complex structure defined by $\mu$. The question is to extend the $s$ operation eq.(28) in order to take these zero modes into account. One may try

$$
s b_{z z}=\Sigma c_{i} \phi^{i}
$$

but $\phi^{i}$ depends on $\mu$ and there is no way to achieve $s^{2} b_{z z}=0$. A nilpotent s operation can be constructed by noticing that there are also fermionic zero modes $s \phi^{i}$. The following s operation is nilpotent:

$$
\begin{aligned}
& s b_{z z}=\sum c_{i} \phi^{i}+d_{i} s \phi^{i} \\
& s d_{i}=-c_{i} \quad s c_{i}=0
\end{aligned}
$$

It is not quite clear whether it is reasonable to use (46) as a basis for gauge fixing.

To conclude, I would say that not much is known about situations where a non involutive distribution is involved, e.g. can it be viewed as the restriction of an 
involutive one? (The auxiliary field problem). Further knowledge is clearly needed!

\section{ACKNOMEDGEMENTS}

I wish to thank $C$. Becchi for detailed informations on his work on strings; M. Tonin for communicating his interpretation of the Batalin Vilkovisky generalization and allowing me to report on it here; L. Baulieu and M. Henneaux for stimulating my interest in this subject; L. Bonora and C. Reina for pointing out the use of projective connections in glueing anomalies on Riemann surfaces.

I also wish to thank the organizers of this meeting for their kind invitation and warmest welcome.

\section{REFERENCES}

[1] E. Cartan, Leçons sur les invariants intégraux, Hermann Paris (1971) (1ere ed. 1922), Les systèmes différentiels extérieurs et leurs applications géométriques, Hermann Paris (1971) (1ere ed. 1945, Act, Sci. Ind. ne $994)$.

[2] L.D. Faddeev, V.N. Popov, Phys. Lett. 25b, 29 (1967).

[3] A.A. Slavnov, Theor. Math. Phys. (TMФ) 10, 153 (1972).

[4] E.S. Fradkin, G.A. Vilkovisky, Phys. Lett. 55B, 224 (1975), CERN report TH.2332 (1977);

I.A. Batalin, G.A. Vilkovisky, Phy. Lett. 69B, 309 (1977).

M. Henneaux, Phys. Rep. 126, 1-66 (1985);

B. Kostant, S. Sternberg, Ann. Phys. 175, (1987);

M. Dubois-Violette, Ann. Inst. Fourier, 1987, to appear.

[5] M.B. Green, J.H. Schwarz, E. Witten, Superstring Theory vol.1, Cambridge University Press (1987);

D. Friedan, E. Martinec, S. Shenker, Nucl. Phys. B271 93 (1986).

[6] J.L. Koszul, Bull Soc. Math. France, 78, 65 (1950).

[7] T. Regge in Relativity Groups Topology II, Les Houches 1983, session XL, B.S. de Witt, R. Stora eds, North Holland 1984;

L. Castellani, R. d'Auria, P. Fré : Supergravity, a geometric perspective, World Scientific, Singapore, to appear;

S. Boukraa, The BRS algebra of a free minimal differential algebra, Nucl. Phys. B. to appear.

[8] L. Baulieu, J. Thierry-Mieg, Nucl. Phys. B288, 259 (1983);

L. Baulieu, Phys. Lett. 126B, 455, (1983);

L. Baulieu, J. Thierry-Mieg, Phys. Lett. 145B, 53 (1984);

L. Baulieu, M. Bellon, Phys. Lett. 161B, 96 (1985). 
[9] A. Rouet, R. Stora, Models with renormalizable Lagrangians: perturbative approach to symmetry breaking, Enseignement du 3ème cycle de la Physique en Suisse romande, pp 47-50, 61-63, June 1973.

The computational appendix pp 61-63 acknowledges $C$. Itzykson for generous help.

This memory goes with another one: the classical paper of I.A. Batalin and G.A. Vilkovisky refers to my "advice to verify the supersymmetry of the theory". Actually, when the first paper by E.S. Fradkin and G.A. Vilkonisky came out, Claude Itzykson and I conjectured over the telephone that their action was invariant under a Slavnov symmetry. The next day, Claude Itzykson called me back to give the formula, which I immediately forgot.

[10] This remark was made by C. Becchi and A. Rouet in January 1974. The hard work of pushing the Slavnov symmetry through renormalization, proving gauge independence and unitarity resulted into:

C. Becchi, A. Rouet, R. Stora, Phys. Lett. 52b, 344 (1974), Comm. Math.

Phys. 42, 127 (1975), Ann. Phys. 98, 287 (1976);

C. Becchi, Comm. Math. Phys. 39, 329 (1975);

G. Bandelloni, C. Becchi, A. Blasi, R. Collina, Ann. IHP XXVIII, 225 (1978), 255 (1978);

The symmetry was found independently by I.V. Tyutin, Lebedev Institute preprint $n^{2} 39,1975$, who did not publish it because some of our work was already published.

[11] C. Becchi, A. Rouet, R. Stora in Renormalization Theory, Erice 1976, G. Velo, A.S. Wightman eds., Reidel NATO ASI Series C Vol.23, 1976.

[12] J.H. Lowenstein, B. Schroer, Phys. Rev. D6, 1553 (1972); K. Symanzik, Lectures in Lagrangian Quantum Field Theory (Islamabad 1968), DESY report T-71/1 (1971).

[13] N.K. Nielsen, Phys. Lett. 103B, 197 (1981);

R.E. Kallosh, Nucl. Phys. B141, 141 (1978);

F.R. Ore, P. van Nieuwenhuizen, Phys. Lett. 112B, 364 (1982).

[14] J. Zinn Justin in Trends in Elementary particle theory, Bonn (1974), H. Rollnick, K. Dietz Eds, Lecture Notes in Physics, Vol.37, Springer Verlag 1975.

[15] J. Wess, B. Zumino, Phys. Lett. 37B, 95 (1971).

[16] C. Becchi, A. Rouet, R. Stora in Field Theory Quantization and Statistical Physics, E. Tirapegui ed., Reidel 1981. The corresponding preprint is dated 1975. We finally decided to publish it because of the theorem in Appendix $D$.

[17] T. Kugo, I. Ojima, Supplement of the progress of theoretical physics 66, 1979.

[18] F.R. Ore, P. Van Nieuwenhuizen, Nucl. Phys. B204. 317 (1982);

L. Baulieu, B. Grossmann, R. Stora, Phys. Lett. 180B, 55 (1986).

[19] L. Brink, P. di Vecchia, P.S. Howe, Phys. Lett. 658, 471 (1976);

S. Deser, B. Zumino, Phys. Lett. 65B 396 (1976);

A.M. Polyakov, Phys. Lett. 103B, 207 (1981);

L. Baulieu, C. Becchi, R. Stora, Phys. Lett. 180B, 55 (1986). 
[20] M. Kato, K. Ogawa, Nucl. Phys. B212, 443 (1983);

R. Marnelius, Nucl. Phys. B211, 14 (1983);

S. Hwang, Phys. Rev. D28, 2614 (1983);

C. Becchi, On the covariant quantization of the free string : the conformal structure, to appear in Nucl. Phys. B.;

L. Baulieu, M. Bellon, Phys. Lett. 196B, 142 (1987);

L. Baulieu, M. Bellon, R. Grimm, Phys. Lett. 198B, 343 (1987).

[21] L. Baulieu, M. Bellon, Phys. Lett I96B, 142 (1987); J.P. Ader, J.C. Wallet, Phys. Lett 192B, 103 (1987),

J.P. Ader, J.C. Wallet, to be publistred.

[22] D. Sullivan, IHES Pub, ne 47 , p.269 ( $j 9.77)$

[23] Symposium on Anomalies, Geómetry, Topology, Argonne Chicago 1985, W.A. Bardeen, A.R. White Eds, World scientific Singapore 1985;

M. Dubois Violette, M. Falon, C.M. Viallet, Comm. Math. Phys. 102, 105 (1985);

J. Mañes, R. Stora, B. Zumino, Comm. Math. Phys. 102, 157 (1985);

L. Alvarez Gaumé, P. Ginsparg, Ann. Phys. 161, 423 (1985);

L. Alvarez Gaumé in Erice 1985, G. Velo, A.S. Wightman Eds. Reidel 1986.

L. Bonora, P. Cotta Ramusino, M. Rinaldi, J. Stasheff, CERN-TH.4647/87$4750 / 87$.

[24] I.M. Singer in Colloque Elie Cartan, Lyon 1984, Asterisque 1985.

[25] D. Gross, R. Jackiw, Phys. Rev. D6, 477 (1972).

[26] G. Bandelloni, private communication.

[27] D. Friedan, Ann.Phys. 163, 318 (1985);

C. Becchi, talk at the Ringberg Workshop 1987, P. Breitentohner, D. Maison, K. Sibold eds, to appear.

[28] K. Stelle, P.C. West, Phys. Lett. Z4B, 330 (1978); Nucl. Phys. B140, 285 (1978);

S. Ferrara, M.T. Grisaru, P. van Nieuwenhuizen, Nucl. Phys. b138, 430

(1978);

L. Baulieu, M. Bellon, Phys. Lett 161B, 96 (1985);

L. Baulieu, M. Bellon, Nucl. Phys. B266, 75 (1986);

L. Baulieu, M. Bellon, Phys. Lett 169B, 59 (1986);

L. Baulieu, M. Bellon, R, Grimm, Nucl. Phys. B. (1987).

[29] B. de Wit, J. van Holten, Phys. Lett. 79B, 389 (1979);

I.A. Batalin, G.A. Vilkovisky, Phys. Lett. 102B, 27 (1981);

I.A. Batalin, Nucl. Phys. B234, 106 (1984); J. M. P. 26, 172 (1985);

Phys. Rev. D28, 2567 (1983);

I.V. Tyutin, Sh.M. Shwartsman, Phys. Lett. 1698, 225 (1986).

[30] R.C. Gunning, Lectures on Riemann surfaces, Princeton Mathematical Notes, Princeton University Press (1966), Princeton N.J.. I thank L. Bonora and C. Reina for suggesting the use of a projective connection in order to get a form of the anomaly valid on an arbitrary Riemann surface. 